July 1994

IFP-708-UNC

VAND-TH-94-15

\title{
Massive Superstrings Are Black Holes
}

\author{
Paul H. Frampton ${ }^{(a)}$ and Thomas W. Kephart ${ }^{(a, b)}$ \\ (a) Institute of Field Physics, Department of Physics and Astronomy, \\ University of North Carolina, Chapel Hill NC 27599-3255 \\ (b) Department of Physics and Astronomy, \\ Vanderbilt University, Nashville, TN 37235*
}

\begin{abstract}
We argue that it is inconsistent to ignore the gravitational backreaction for on-shell superstring states at the Planck mass and beyond, and that these quantum states become Kerr black holes in the classical limit. Consequences are discussed.
\end{abstract}

*Permanent address 
In assessing progress towards a satisfactory theory of quantum gravity it is important to evaluate the present status of, and relationship between, the principal disparate yet overlapping approaches: (i) the operator, canonical and path integral approaches [1] are the most direct and historically first attempts at quantum gravity based on techniques used so successfully for quantum field theory in flat spacetime; (ii) the dilemmas and paradoxes surrounding black hole evaporation and thermodynamics, including backreaction, provide a fruitful direction toward quantum gravity [2]; (iii) superstring theory in some version yet to be identified promises a basis for quantum gravity and its connection to particle theory [3], [4]; and (iv) the ideas within quantum cosmology about a satisfactory boundary condition to characterize the big bang provide an equally valid mode of investigation[5].

Here we shall concentrate on the relationship between (ii) and (iii), black holes and superstrings and their thermodynamics at the Planck scale. Toward the end of the Letter we shall comment about their impact on (iv), the preinflationary big bang.

The string spectrum as determined by factorization of the tree amplitudes comprises, to lowest order, an infinite set of parallel linear Regge trajectories (Fig. 1). The leading quantum trajectory for the superstring is [4]

$$
\alpha_{\text {graviton }}(s)=2+\alpha^{\prime} s
$$

with other trajectories at

$$
\alpha(s)=2+\alpha^{\prime} s-n / 2
$$

for $n=1,2, \ldots$. The massless spectrum comprises the graviton, the gravitino, and lower spin states, e.g., gauge bosons, quarks and leptons.

Massive states have masses at least comparable to the Planck mass $\sim 10^{-19} \mathrm{GeV}$. For the leading trajectory (1), $J \sim \alpha^{\prime} M^{2}$ as $M^{2} \rightarrow \infty$. What is the slope $\alpha^{\prime}$ ? For the leading trajectory a classical model is of a straight string of length $2 l$ and density $\rho$ per unit length. Using the boundary condition that the ends move at the speed of light $(c)$ the value of $J$ is

$$
\int_{-l}^{l} d x \rho(x c / l) x=c M^{2} / 6 \rho
$$


and $\rho$ is the ratio $($ PlanckMass $) /($ PlanckLength $)=(\hbar c / G)^{1 / 2}\left(\hbar G / c^{3}\right)^{-1 / 2}=c^{2} / G$. Thus the superstring slope is roughly

$$
\alpha_{\text {superstring }}^{\prime}=G / 6 c
$$

In scattering processes it is possible to show that the states with $J \leq\left(M^{2} \alpha^{\prime}\right)^{1 / 2}$ are significantly more strongly coupled [6] although all states with $J \leq M^{2} \alpha^{\prime}$ do occur.

It is interesting to compare the string slope, eq (4) to the condition relating $J$ and $M$ for a Kerr black hole [7]. The Kerr solution without charge has an event horizon at radius

$$
R(\text { Kerr })=G M / c^{2}+\sqrt{G^{2} M^{2} / c^{4}-J^{2} / M^{2} c^{2}}
$$

in $d=4$ spacetime dimensions for angular momentum $\hbar J$. Only states with

$$
J \leq G M^{2} / c
$$

may form a black hole. Thus all on-shell superstring states below the line

$$
\alpha=G M^{2} / c
$$

can and will collapse to black holes. This is certainly true in the classical limit where the black hole mass satisfies $M_{\text {Planck }} \ll \mu$; it is not clear where or if the argument breaks down for $\mu \rightarrow M_{\text {Planck. }}$ Comparing Eqs. (1) and (7) shows that one expects all massive superstring states on mass shell to form black holes. The Chew-Frautschi plot is depicted in Fig. 1. In Eqs. (1), (2), and in Fig. 1 backreaction can shift the black hole masses giving some non-linearity to the Regge tragectories; this merits further study using the results of [8].

The superstring massive states are physical black holes only on mass shell; off-shell, as virtual states contributing to low-energy scattering of massless particles, this does not detract from the usefulness of perturbative superstrings as an effective theory.

Accepting that massive superstring states describe black holes, we may use a toy dual model for scattering of two black holes. It was shown twenty-five years ago [6] that in a dual model for scattering two states the biggest contribution comes from those states with 
angular momentum $J \leq$ (constant) $M$ as one would expect from a lever-arm argument for a given impact parameter. There, one started with a hadronic amplitude

$$
A(s, t)=\Gamma(1-\alpha(s)) \Gamma(1-\alpha(t)) / \Gamma(1-\alpha(s)-\alpha(t))
$$

and computed the asymptotic form of the partial wave amplitudes; we shall go into more details below, where we shall assume Eq. (8) is applicable to spinless black-hole scattering.

A connection between superstrings states and black holes can be made in a different fashion. The Helmholtz free energy per unit 4-volume F/VT in a thermal distribution of strings at temperature $\mathrm{T}$ can be evaluated from world- sheet path integrals for string propagation at the one-loop level [9] for $\mathrm{T}$ below the value $T_{\text {critical }}$ (not very different, but somewhat below, $T_{\text {Hagedorn }}$ and $\left.T_{\text {Planck }}\right)$ where a phase transition may occur [10]. Above $T_{\text {critical }}$ a zero-loop contribution dominates $\mathrm{F}$ such that even the concept of temperature necessarily becomes ill-defined because gravitational instability disallows consideration of a suitable large volume limit.

The free energy from loop contributions and also from $1 / \mathrm{N}$ estimates give, at fixed effective coupling $g_{\text {eff }}^{2}$, the dependence [10]

$$
F / V T \sim T^{p}
$$

where $p=(d-1)$ for field theory in d spacetime dimensions, and $p=0$ and 1 for the cases of open and closed strings respectively. This is how it was suprisingly concluded that strings above the Hagedorn temperature have far fewer degrees of freedom than a corresponding field theory. To compare this superstring free energy to that calculated for black holes, we cite the generalized Helmholtz free energy [11] for the spinless (Schwarzschild) limit. This result rewritten for a dilute gas of black holes is that

$$
F=-4 \pi M^{2} T
$$

which can be rewritten as

$$
F / V T=-1 / 16 \pi T_{H}^{2} V
$$


so that for fixed-size black holes the free energy per unit 4-volume corresponds (in first approximation) to a constant value independent of the temperature of the surroundings; that is, $p=0$ in Eq. (8). This behavior is similar to that of the open string. It is interesting to note that for closed strings [10] where $p=1$ there is a mysterious ultra-violet pathology in the classical limit $\hbar \rightarrow 0$; it will be interesting to investigate whether this smooth limit obtaining for open strings carries over to their Kerr counterparts.

Alternatively, by setting $T_{H}=T$ in Eq. (10), we find

$$
F / V T \sim T^{-2}
$$

with an $\hbar^{-2}$ ultra-violet divergence in the classical limit. For comparison purposes it would be interesting to have a calculation of free energy for a thermal distribution of membranes.

If one accepts our thesis that massive superstrings on-shell are Kerr black holes, they must surely be quantum black holes. This implies that the black hole entropy should be computed along the general lines presaged in [12], [13]. Our result that massive superstrings are black holes may be interestingly compared to work of 't Hooft [14] who gave the complementary argument that black holes are strings.

The spectrum of quantum black holes is predicted by the superstring to be $\sqrt{n} M_{\text {Planck }}$ where $n$ is an integer. This immediately provides predictions for the spectrum of emitted massless particles, for the entropy, and for the evaporation.

Suppose a black hole of mass $\sqrt{n} M_{\text {Planck }}$ radiates a photon in decaying to a smaller black hole of mass $\sqrt{n^{\prime}} M_{\text {Planck. }}$. (A similar analysis could be carried out for decay to other massless states [15].) Then the energy of the photon in the rest frame of the initial black hole is easily shown to be

$$
E_{\gamma}=\left(M_{\text {Planck }} / 2\right)\left(n-n^{\prime}\right) / \sqrt{n}
$$

This implies a characteristic spectrum of radiation, for example

$$
2 E_{\gamma} / M_{\text {Planck }}=k_{1} / \sqrt{n}, k_{2} / \sqrt{n-k_{1}}, \ldots, k_{n} / \sqrt{n-\sum_{i=1}^{n-1} k_{i}}
$$


where the $k_{i}$ are integers. For $n \rightarrow \infty$ this approximates a continuous spectrum, but for small $\mathrm{n}$ the quantum nature becomes apparent and each photon has of order the Planck energy in the rest frame of the decaying black hole [16]. The entropy is defined as the logarithm of the number of different quantum configuration of the black hole. As can be seen from Eq. (14), this corresponds to the number of ordered partitions of $n$, that is $2^{n-1}$ , and consequently an entropy

$$
S=(n-1) \ln 2
$$

This agrees with [13].

To summarize, all mass-shell elementary particles are either massless or black holes. The massless states are the superstring zero modes and include all the familiar particles of the standard model, and possibly further particles massless with respect to the Planck scale. The black holes are quantum states with spectrum $M(n)=\sqrt{n} M_{\text {Planck }}$ (up to backreaction corrections), and are gravitationally confined superstrings.

Let us pursue further the scenario to which we have been led. During the very early universe these mini black holes can either evaporate into massless states or, if the collsion time is sufficiently short compared to the Hubble time and to the evaporation lifetime, they can also coalesce into successively larger black holes.

Returning to the toy dual model for black hole scattering, Eq. (8), we may partial wave project the residue $F_{n}(t)$ at the level $\alpha(s)=n$ for Regge intercept $a$ and external mass $\mu$ by writing

$$
C_{n}^{l}=(l+1 / 2) \int_{-1}^{+1} F_{n}(t(z)) P_{l}(z) d z
$$

and hence

$$
C_{n}^{l}=n(l+1 / 2)(2 \pi)^{1 / 2}\left[\left(n-a-4 \mu^{2}\right) / 2\right]^{-1 / 2} J
$$

in which

$$
J=1 / 2 \pi i \int d \zeta / \zeta^{1 / 2} \exp \left[\left(3 a+4 \mu^{2}-1\right) \zeta / 2\right] I_{l+1 / 2}\left[\left(n-a-4 \mu^{2}\right) \zeta / 2\right] /[2 \sinh (\zeta / 2)]^{n+1}
$$


Now by using asymptotic versions for the modified Bessel function for $n, l \rightarrow \infty$ we find that

$$
C_{n}^{l} \sim[1 / 2(l+1 / 2)]^{1 / 2} /\left[\left(\zeta_{0} / 2\right) \cosh \left(\zeta_{0} / 2\right)-\sinh \left(\zeta_{0} / 2\right)\right]^{1 / 2} E
$$

where

$$
E=\left[\exp \left(\cosh \left(\zeta_{0} / 2\right) \tanh \left(\zeta_{0} / 4\right)\right]^{l+1 / 2} /\left[2 \sinh \left(\zeta_{0} / 2\right)\right]^{n} 2 \cosh \left[\left(3 a+4 \mu^{2}-1\right) \zeta_{0} / 2\right]\right.
$$

for $n-l$ even. (For $n-l$ odd the final cosh becomes a sinh.) From this we expect for black hole scattering a dominance by intermediate Schwarzschild-like black holes with $l \leq \sqrt{n}$, and of coalescence into such black holes. The probability for such fusion can be estimated using the black hole lifetime $\tau_{H} \sim M^{3} / n_{\phi}$ and cross-section $\sigma \sim M^{2} / \beta$ where $n_{\phi}$ is the number of distinct massless modes and $\beta$ is the black hole velocity.

If the universe undergoes inflation after the big bang, then the number of primordial black holes remaining today will be diluted to a few per horizon size provided that the early universe was not matter dominated by the black holes to the extent that overclosure and recollapse occurred. We may check from a toy calculation that this dilution occurs, assuming that all black holes started at the Planck time $t_{1}=3 \times 10^{-44} s$ with $\mu=M_{\text {Planck }}$. (It is not clear that there cannot be a spectrum of black hole masses resulting from string winding modes, or a thermal distribution there for $R<L_{\text {Planck }}$ [17].) At $t=t_{1}$ the horizon volume is estimated as $V_{1}=10^{-9} \mathrm{~cm}^{3}$ and critical density is $\rho_{1}=2.5 \times 10^{93} \mathrm{~g} / \mathrm{cm}^{3}$. Since the black hole lifetime varies as $M^{3}$ we take time steps $t_{n}=(8)^{n-1} \times 3 \times 10^{-44} s$ at which $\rho_{n}=(64)^{n-1} \times \rho_{1}$ This approach approximates the quantum nature of mini black holes discussed above. The black hole mass is $\mu_{n}=(2)^{n-1} \times 10^{-5} \mathrm{~g}$ due to successive approximate doublings by fusion with cross-section $\sigma_{n}=(4)^{n-1} \sigma_{1} / \beta_{n}^{2}=(4)^{n-1} \times 10^{-66} \mathrm{~cm}^{3} / \beta_{n}^{2}$. The probability $p_{n}$ of a fusion during the $n^{\text {th }}$ stage is

$$
p_{n}=\sigma_{n} \tau_{n} \beta_{n} \rho_{n} / \mu_{n}
$$

where $\beta_{n}$ is the velocity $\beta_{n}^{2}=(4 \sqrt{2})^{n-1}$ and $\rho_{n}$ is the black hole density $\rho_{n}=\rho_{n-1}(128)^{-1} p_{n-1}$. Thus 


$$
p_{n}=2^{-7 / 4}\left(p_{n-1}\right)^{2}=2^{-a_{n}}
$$

where

$$
a_{n}=2 a_{n-1}+7 / 4=2^{n}(15 / 8)-7 / 4
$$

We find that after seven 8-foldings corresponding to $T \sim 10^{16} \mathrm{GeV}$ all the primordial black holes have evaporated as anticipated. Thus primordial black holes do not survive past the GUT scale. Black holes can reform during cosmological phase transitions [18, but it is again ruled out that they survive evaporation to seed structure formation.

To conclude, superstrings are at best useful as a low-energy effective field theory where the massive black holes are off-shell. Any more complete theory for higher (Planckian) emerges must involve massive superstrings as black holes including their backreaction.

We both thank the Aspen Center for Physics for hospitality while preparing this article. This work was supported in part by the US Department of Energy under Grant Numbers DE-FG05-85ER-40219 and DE-FG05-85ER-40226.

\section{Figure Caption:}

Fig 1. Chew-Frautschi plot showing the first seven Regge trajectories. The line marked by an asterisk $(*)$ is that below which resonances are strongly coupled (see Ref. 6); the line marked by $(* *)$ is the black hole bound. 


\section{REFERENCES}

[1] S. W. Hawking, The Path-Integral Approach to Quantum Gravity, in General Relativity: An Einstein Centenary Survey, Editors S. W. Hawking and W. Israel, Cambridge University Press (1979), Chapter 15.

[2] S. W. Hawking, Comm. Math. Phys. 43, 199(1975).

[3] P. H. Frampton, Dual Resonance Models, Benjamin (1974); reprinted in an extended form as Dual Resonance Models and Superstrings, World Scientific (1986).

[4] M. B. Green, J. H. Schwarz and E. Witten, Superstring Theory, Cambridge University Press (1986).

[5] J. B. Hartle and S. W. Hawking, Phys. Rev. D28, 296(1983).

[6] P. H. Frampton and Y. Nambu, Asymptotic Behavior of Partial Widths in the Veneziano Model of Scattering Amplitudes, in Quanta, Festscrift for G. Wentzel, Editors: P. G. O. Freund, C. J. Goebel and Y. Nambu, University of Chicago Press (1970), page 403.

[7] R. Kerr, Phys. Rev. Lett. 11, 327(1963);

S. Chandrasekhar, The Mathematical Theory of Black Holes, Oxford University Press (1983).

[8] J. W. York, Jr, Phys. Rev. D31, 775(1985);

D. Hochberg and T. W. Kephart, Phys. Rev. D47, 1465(1993);

D. Hochberg, T. W. Kephart and J. W. York, Phys. Rev. D48, 479(1993);

D. Hochberg, T. W. Kephart and J. W. York, Phys. Rev. D49, 5257(1993).

[9] J. Polchinski, Comm. Math. Phys. 104, 37(1986).

[10] J. J. Atick and E. Witten, Nucl. Phys. B310, 291(1988).

[11] J. W. York, Phys. Rev. D33, 2092(1986).

[12] W. H. Zurek and K. S. Thorne, Phys. Rev. Lett 54, 2171(1985). 
[13] V. F. Mukhanov, The Entropy of Black Holes, in Complexity, Entropy and the Physics of Information, SFI Studies in the Science of Complexity, Vol. 8, Editor W. H. Zurek, Addison-Wesley (1990), page 47.

[14] G. 't Hooft, Nucl. Phys. B335, 138(1990).

[15] D. N. Page, Phys. Rev. D13, 198(1976).

[16] A quantum spectrum for black holes/ strings of this type has very interesting implications if some of these objects are present today. Assuming they lose energy through two-body decays, they would produce extremely energetic cosmic rays ( approx. $10^{28}$ $\mathrm{eV}$ ) which could then degrade by scattering from the cosmic background radiation to produce the high energy end of the observed cosmic ray spectrum (approx. $10^{20} \mathrm{eV}$ ), for which there there is at present no conventional physics explanation.

[17] R. Brandenberger and C. Vafa, Nucl. Phys. B316, 371(1989).

[18] P. N. Nasel'skii and A. C. Poharev, Sov. Astron. 29, 487(1985). 